ARQGA / 1100

\title{
INCIDÊNCIA COMPARATIVA DA APENDICITE AGUDA EM POPULAÇÃO MISCIGENADA, DE ACORDO COM A COR DA PELE
}

\author{
Andy PETROIANU, Jose Estevão de OLIVEIRA-NETO e Luiz Ronaldo ALBERTI
}

RESUMO - Racional - Apendicite aguda é uma emergência cirúrgica muito comum, mas sua etiopatogenia ainda per manece incompreendida Objetivo - Avaliar aspectos epidemiológicos da apendicite aguda relacionados à cor da pele, sexo e idade. Métodos - Foram estudados 500 pacientes operados de apendicite aguda e outros 500 doentes aleatórios tratados por diferentes doenças no Hospital das Clínicas da Universidade Federal de Minas Gerais, Belo Horizonte, MG. Avaliaram-se sexo, idade e cor da pele. Os resultados foram comparados por meio do teste qui-quadrado. Resultados - Significativa prevalência de pacientes leucodérmicos $(73,8 \%)$ foi verif icada nessas séries. Não houve predominância por sexo. O número de homens leucodémicos com apendicite aguda foi significativamente maior do que homens leucodérmicos do g rupo-controle. A incidência de melanodérmicos com apendicite foi signif icativamente menor do que a de pacientes negros do grupo-controle. A idade média da apendicite aguda foi de 23,2 anos para os pacientes leucodérmicos e de 32,3 anos para os melanodérmicos. Conclusões - Foi nítida a relação entre apendicite aguda com a cor branca da pele.

DESCRITORES - Apendicite. Pigmentação da pele. Grupos étnicos. Sexo. Grupos etários.

\section{INTRODUÇÃO}

A apendicite aguda é a mais freqüente condição cirúrgica abdominal $^{(14)}$. Essa doença tem sido atribuída à obstrução mecânica, à dieta inadequada de fibras e à susceptibilidade familiar ${ }^{(1,3,6,9,14)}$. Outros fatores relacionados à estrutura populacional, melhora das condições socioeconômicas e patógenos bacterianos, virais ou parasitários, também estão envolvidos na etiopatogenia dessa afecção ${ }^{(1,2,5)}$. Entretanto, sua etiologia e epidemiologia ainda permanecem pouco compreendidas $^{(1,2,5)}$. A relação entre apendicite aguda e cor da pele é um dos aspectos pouco estudados.

Trabalhos realizados em países com predominância de população branca mostraram incidências de apendicite, que alcançaram 17\% na Dinamarca e 12\% na Inglaterra. Todavia, em populações negras de países da África essa doença é muito rara, com incidências que variam entre $0,3 \%$ e $1 \%{ }^{(12,13)}$. Pessoas de origem indígena e de etnia miscigenada desenvolvem apendicite numa taxa de $2 \%$ a $3 \%{ }^{(13)}$. Nos Estados Unidos, a incidência de apendicite aguda é de 1,6\%, com predominância para a cor branca ${ }^{(1)}$.
Na literatura, a epidemiologia da apendicite em diferentes raças está relacionada não só com a cor da pele, mas também com fatores genéticos, poluentes ambientais e hábitos dietéticos ${ }^{(3,9)}$. Brancos e negros de diferentes regiões de um mesmo país apresentam incidências de apendicite aguda variadas $^{(4,13)}$.

Os brasileiros são uma mistura de várias raças. Os brancos são provenientes de diversos países europeus, principalmente Portugal, Itália e Alemanha. Os negros são descendentes de países da África Ocidental, destacando-se Angola, Moçambique e Nigéria. Os poucos indígenas estão reunidos em pequenos grupos localizados em diversas regiões, especialmente no norte do país. Entretanto, mais de um terço da população brasileira é composta pela mistura de várias raças $^{(12,13)}$. Essa origem mesclada de nossa população impossibilita considerações étnicas, porém é pertinente a classificação de acordo com a cor da pele.

A localização do Estado de Minas Gerais faz com que ele seja o mais representati vo no que concerne à distribuição populacional por cor de pele. Em estudo epidemiológico prévio, verificou-se que o padrão populacional dos pacientes

Trabalho realizado no Departamento de Cirurgia da Faculdade de Medicina da Universidade Federal de Minas Gerais.

Endereço para correspondência - Dr. Andy Petroianu - Av.Afonso Pena, 1626 - ap. 1901 - 30130-005 - Belo Horizonte, MG. E-mail: petroian@medicina.ufmg.br 
do Hospital das Clínicas (HC) não é diferente do encontrado em todo o Estado de Minas Gerais. Por conse guinte, é pertinente supor que uma amostra de pacientes operados por apendicite aguda nesse hospital corresponda à população do Estado de Minas na proporção de cor da pele.

Por causa da particularidade da população brasileira e a sua representatividade no $\mathrm{HC}$, considerou-se pertinente verificar aspectos epidemiológicos da apendicite aguda em amostra populacional miscigenada.

\section{MÉTODO}

O presente estudo foi aprovado por Comissão de Ética do Departamento de Cirurgia da Faculdade de Medicina da Universidade Federal de Minas Gerais, de acordo com as recomendações da Declaração de Helsinque e a Resolução no 196/96 do Ministério da Saúde sobre pesquisa envolvendo seres humanos.

Este trabalho retrospectivo avaliou 500 prontuários de pacientes operados por apendicite aguda no HC da Universidade Federal de Minas Gerais de 1992 a 1996. Foram pesquisados idade, se xo e cor da pele (leucodérmicos, feodérmicos, melanodérmicos), de acordo com as informações contidas nos prontuários. Estudos histopatológicos confirmaram a apendicite aguda em todos os casos. Pessoas submetidas a apendicectomia com apêndice histologicamente normal não foram consideradas.

Prontuários de outros 500 pacientes tratados por diferentes doenças crônicas e não inflamatórias ou traumáticas, no mesmo hospital em 1996 foram aleatoriamente estudados nos mesmos parâmetros dos apendicectomizados.

Foram excluídos os pacientes que não se enquadraram perfeitamente na proposta metodológica desta pesquisa ou quando houve qualquer dúvida relativa aos parâmetros estudados. Não houve repre- sentante da população indígena na casuística deste trabalho, tanto dos apendicectomizados quanto do grupo-controle. Os resultados foram comparados por meio do teste qui-quadrado. O desvio padrão foi calculado para todas as variáveis em estudo. As diferenças foram consideradas significativas para valores correspondentes a $P<0,05$.

\section{RESULTADOS}

A Tabela 1 mostra as características epidemiológicas dos 500 pacientes com apendicite e dos 500 pacientes operados por doenças diferentes (grupo-controle). A incidência de apendicite foi ligeiramente maior no sexo masculino, mas a diferença não foi significativa. Não houve diferença entre as idades de homens e mulheres com apendicite quanto à cor da pele. A comparação entre as idades de pacientes com apendicite com diferentes cores da pele (leucodérmicos $23,2 \pm 14,4$; feodérmicos 24,9 $\pm 16,7$ e melanodérmicos $32,3 \pm 16,7$ ) não foi significativa devido ao desvio padrão da média, entretanto, a média das idades dos melanodérmicos foi maior.

No grupo-controle, não houve diferença signif icativa entre homens e mulheres, quanto a idade e cor da pele. A proporção de cor da pele nesse grupo não foi diferente da mesma proporção encontrada no Estado de Minas Gerais (leucodérmicos 57\%, feodér micos $34 \%$ e melanodérmicos $8 \%)^{(11)}$. Apendicite aguda foi encontrada em nove desses pacientes: seis homens e três mulheres. As idades variaram entre 16 e 73 anos. Sete desses pacientes eram leucodérmicos e dois feodérmicos.

A prevalência de leucodérmicos com apendicite aguda foi signif icativamente maior no grupo estudado, do que no grupo-controle e na população de Minas Gerais ${ }^{(11)}$. A incidência de pacientes negros com apendicite aguda foi signif icativ amente menor, em comparação ao grupo-controle e à população de Minas Gerais ${ }^{(11)}$.

TABELA 1 - Características dos 500 pacientes operados por apendicite aguda e 500 pacientes tratados por diferentes doenças (controle)

\begin{tabular}{|c|c|c|c|c|c|c|}
\hline & \multicolumn{2}{|c|}{ Homens } & \multicolumn{2}{|c|}{ Mulheres } & \multicolumn{2}{|c|}{ Total } \\
\hline № de pacientes & 279 & 209 & 221 & 291 & 500 & 500 \\
\hline $\begin{array}{l}\text { Idade (anos) } \\
\text { limites } \\
\text { média }\end{array}$ & $\begin{array}{c}2-84 \\
25,5 \pm 14,8\end{array}$ & $\begin{array}{c}3-96 \\
32,6 \pm 20,1\end{array}$ & $\begin{array}{c}4-78 \\
28,0 \pm 16,0\end{array}$ & $\begin{array}{c}3-93 \\
38,6 \pm 24,3\end{array}$ & $\begin{array}{c}2-84 \\
26,6 \pm 15,4\end{array}$ & $\begin{array}{c}3-96 \\
35,0 \pm 22,3\end{array}$ \\
\hline
\end{tabular}

- Diferentes entre o grupo com apendicite e o grupo-controle relativos ao mesmo parâmetro $(P<0,05)$ 


\section{DISCUSSÃO}

Os resultados desta série confirmam relatos da literatura em relação à epidemiologia da apendicite aguda em várias populações ${ }^{(10,12)}$. Essa doença ocorre mais em pessoas jovens, sem prevalência de sexo, tendo por idade média 21 anos na população geral ${ }^{(1)}$. Entretanto, é importante ressaltar que a idade encontrada em pessoas negras da presente casuística, foi superior à evidenciada para os pacientes de outras cores de pele e à estabelecida na literatura como limite de ocor rência da apendicite, que é de 28 anos.

Parece haver fatores, talvez genéticos, relacionados à cor da pele que são responsáveis pela prevalência da apendicite aguda em pessoas leucodérmicas. As diferenças entre as cores de pele não podem ser atribuídas a alguma particularidade do $\mathrm{HC}$, que é referência para pessoas de todas as classes sociais. Por tanto, os resultados encontrados na presente investigação podem ser devidos à relação entre cor da pele e apendicite aguda, como mencionado em outros estudos ${ }^{(1,4,5,10,13)}$. Deve-se, entretanto, ressaltar que os dados de prontuário podem deixar dúvida quando ao paciente feodérmico de tonalidade mais escura e o melanodérmico. Essa diferença é muito difícil de ser estabelecida no Brasil, porém todos os dados estatísticos englobam ambos os g rupos de pessoas como melanodér micos ${ }^{(11)}$.

Considerando a predisposição familiar da apendicite, bem descrita na literatura, essa relação também deve ser levada em conta. Fatores ambientais poderiam explicar apendicite aguda em populações dife- rentes, mas esse parâmetro não interferiu nos resultados deste trabalho e não foi objetivo de estudo na presente pesquisa ${ }^{(2,14)}$.

Nesta série, não se avaliou a dieta dos pacientes, que é assunto ainda controverso na literatura com respeito à apendicite. Alguns autores sugerem que uma dieta pobre em fibras possa ser responsável pela doença ${ }^{(3,9)}$. Entretanto, outros não encontraram relação entre hábitos alimentares e apendicite ${ }^{(6)}$. Levando-se em conta que os brasileiros tendem a consumir dietas ricas em fibras, independentemente de classe social ou cor da pele, pode-se supor que aspectos dietéticos não explicam a incidência variável de apendicite aguda em pessoas de diferentes cores de pele.

\section{CONCLUSÃO}

A apendicite aguda é uma doença que predomina em jovens de ambos os sexos, porém parece haver tendência dela ser menos freqüente e ocorrer em faixa etária maior nas pessoas melanodérmicas. Esses achados precisam ser melhor estudados, principalmente com vista a particularidades morfológicas do apêndice relacionadas à cor da pele.

\section{AGRADECIMENT OS}

Os autores agradecem ao CNPq e FAPEMIG pelos auxílios financeiros que per mitiram a realização deste trabalho.

Petroianu A, Oliveira-Neto JE, Alberti LR. Comparative incidence of acute appendicitis in a mixed population, related to the skin color. Arq Gastroenterol 2004;41(1):24-26.

ABSTRACT - Background - Acute appendicitis is a very common surgical emergency, but its etiology and pathology remain incompletely understood. Objective - To elucidate epidemiological aspects of acute appendicitis related to skin color, gender and age.Methods - Five-hundred patients operated on for acute appendicitis, and other 500 patients treated for different diseases and considered as a control g roup were studied at "Hospital das Clínicas", Federal University of Minas Gerais, Belo Horizonte, MG, Brazil. Gender, age and skin color were investigated. The results were compared by chi-square test. Results - There was no prevalence related to gender. A significant prevalence of white patients (73.8\%) was verif ied in this series. The number of white men with appendicitis was significantly higher than white men in the control group. The incidence of black patients with appendicitis was significantly lower than black patients of the control group. The mean age was 23.2 years old for the white patients with appendicitis and 32.3 years old for the black patients. Conclusions - The present paper shows an e vident relation between appendicitis and white skin color.

HEADINGS - Appendicitis. Skin pigmentation. Ethnic groups. Sex. Age groups.

\section{REFERÊNCIAS BIBLIOGRÁFICAS}

1. Addiss DG, Shaffer N, Foewler BS, Taux e RV. The epidemiology of appendicitis and appendectomy in the United States. Am J Epidemiol 1990;132:910-25.

2. Anderson N, Griffiths H, Murphy J. Is appendicitis familial? Br Med J 1979;ii:697-8.

Arnbjörnsson E. Acute appendicitis and dietary fiber. Arch Surg 1983;118:868-70. Ashley DJB. Observations on the epidemiology of appendicitis. Gut 1967;8:533-8 Attwood SEA, Caf ferke y MT, West AB. High appendicectomy rates in Ireland. J Epidemiol Community Health 1987;41:72-3.

6. Barker DJP. Acute appendicitis and dietary fibre. Br J Med J 1985;290:1125-7

7. Barker DJP, Osmond C, Golding J. Acute appendicitis and bathrooms in three samples of British children. Br Med J 1988;296:956-8.
8. Brumer M. Appendicitis. Br J Surg 1970;57:93-9.

9. Burkitt DP. The etiology of appendicitis. Br J Surg 1971;58:695-9.

10. Jess P. Acute appendicitis. Scand J Gastroenterol 1983;18:161-3.

11. IBGE-SEI/SEPLAN-MG. Censo demográfico do Estado de Minas Gerais Anuário Estatístico do Brasil 1997; 57:13-57.

12. WalkerAR, Walker BF. Appendectomy in South African inter-ethnic school pupils. Am J Gastroenterol 1987;82:219-22.

13. Walker AR, Walker BF, Mantesi B. Appendicitis in Soweto, South Africa. J R Soc Health 1989;109:190-2.

14. Wolkomir A, McGovern P. Seasonal variation of acute appendicitis. South Med J 1987;80:958-60.

Recebido em 25/4/2003 Aprovado em 15/8/2003 